\title{
Heisenberg-Fisher thermal uncertainty measure
}

\author{
F. Pennini and A. Plastino \\ Instituto de Física La Plata (IFLP), Universidad Nacional de La Plata (UNLP), and Argentine National Research Council (CONICET), \\ C.C. 727, 1900 La Plata, Argentina
}

(Received 1 December 2003; revised manuscript received 2 February 2004; published 21 May 2004)

\begin{abstract}
We establish a connection among (i) the so-called Wehrl entropy, (ii) Fisher's information measure $I_{\beta}$, and (iii) the canonical ensemble entropy for the one-dimensional quantum harmonic oscillator (HO). We show that the contribution of the excited HO spectrum to the mean thermal energy is given by $I_{\beta}$, while the pertinent canonical partition function is essentially given by another Fisher measure: the so-called shift invariant one. Our findings should be of interest in view of the fact that it has been shown that the Legendre transform structure of thermodynamics can be replicated without any change if one replaces the Boltzmann-GibbsShannon entropy by Fisher's information measure [B. R. Frieden, A. Plastino, A. R. Plastino, and H. Soffer, Phys. Rev. E 60, 48 (1999)]. Fisher-related uncertainty relations are also advanced, together with a Fisher version of thermodynamics' third law.
\end{abstract}

DOI: 10.1103/PhysRevE.69.057101

PACS number(s): 05.70.-a, 03.67.-a, 05.20.-y

\section{INTRODUCTION}

We will here explore some features of an informationtheoretic uncertainty measure, the Wehrl entropy [1] $I_{W}$. As shown by Lieb [2], $I_{W} \geqslant 1$, and this bound represents a strengthened version of the uncertainty principle. In the case of a harmonic oscillator in a thermal state, $I_{W}$ coincides with the logarithmic information measure of Shannon's in the high-temperature regime. However, it does not vanish at zero temperature, thus supplying a nontrivial measure of uncertainty due to both thermal and quantum fluctuations [3]. It will be shown here that intriguing connections link $I_{W}$ to Fisher's information measure $I$. As far as possible, we will use the notation of Anderson and Halliwell [3].

The present endeavor is motivated by the fact that much interesting work has recently been devoted to the physical applications of Fisher's information measure (FIM) (see, for instance, Refs. [4-8]). Frieden and Soffer [4] have shown that Fisher's information measure provides one with a powerful variational principle - the extreme physical information one, which yields most of the canonical Lagrangians of theoretical physics [4,5]. Additionally, $I$ has been shown to provide an interesting characterization of the "arrow of time," alternative to the one associated with Boltzmann's entropy $[9,10]$. Thus, unraveling the multiple FIM facets and their links to physics should be of general interest. The Legendre transform structure of thermodynamics can be replicated as well, without any change, if one replaces the BoltzmannGibbs-Shannon entropy $S$ by Fisher's information measure $I$. In particular, (i) $I$ possesses the all important concavity property [7], and (ii) use of the Fisher's measure allows for the development of a thermodynamics that seems to be able to treat equilibrium and nonequilibrium situations in a manner entirely similar to the conventional one [7]. Here, the focus of our attention will be, following Ref. [3], the thermal description of harmonic oscillator (HO).

For the convenience of the reader, in Sec. II we review some fundamental aspects of the HO canonical-ensemble description from a coherent states' viewpoint [3]. We also discuss some ideas related to Fisher's information measure, the protagonist of the present effort, in Sec. III. In Sec. IV we explore its properties with regards to temperature's estimation while, in Sec. V, we establish some results concerning uncertainty relations. Finally, we draw some conclusions in Sec. VI.

\section{BACKGROUND NOTIONS}

In Ref. [3] the authors discuss quantum-mechanical phase-space distributions expressed in terms of the celebrated coherent states $|z\rangle$ of the harmonic oscillator, eigenstates of the annihilation operator $\hat{a}[11,12]$ :

$$
\begin{aligned}
& \hat{H}_{o}=\hbar \omega\left[\hat{a}^{\dagger} \hat{a}+1 / 2\right], \quad \hat{a}=i(2 \hbar \omega m)^{-1 / 2} \hat{p}+(m \omega / 2 \hbar)^{1 / 2} \hat{x} ; \\
& 2 z=\left(x / \sigma_{x}+i p / \sigma_{p}\right), \\
& z=(m \omega / 2 \hbar)^{1 / 2} x+i(2 \hbar \omega m)^{-1 / 2} p, \equiv x^{\prime}+i p^{\prime} \quad \text { with } \quad x^{\prime} \\
& =x / 2 \sigma_{x}, \quad p^{\prime}=p / 2 \sigma_{p} ; \\
& \sigma_{x}=(\hbar / 2 m \omega)^{1 / 2}, \quad \sigma_{p}=(\hbar m \omega / 2)^{1 / 2}, \quad \sigma_{x} \sigma_{p}=\hbar / 2 .
\end{aligned}
$$

Variances $\sigma$ are evaluated for the $\mathrm{HO}$ ground state. Coherent states span Hilbert's space, constitute an overcomplete basis, and obey the completeness rule (use "natural variables" $\left.x^{\prime}, y^{\prime}\right)[11]$ :

$$
\begin{gathered}
\int \frac{d^{2} z}{\pi}|z\rangle\left\langle z\left|=\int \frac{d x d p}{2 \pi \hbar}\right| x, p\right\rangle\langle x, p|=1, \\
d^{2} z=d \operatorname{Re}(z) d \operatorname{Im}(z)=\frac{d x d p}{2 \hbar} \equiv d x^{\prime} d p^{\prime} .
\end{gathered}
$$

Variances $\sigma$ are evaluated for the HO ground state. The Wehrl entropy [1] is defined as 


$$
I_{W}=-\int \frac{d p d x}{2 \pi \hbar} \mu(x, p) \ln \mu(x, p),
$$

where $\mu(x, p)=\langle z|\hat{\rho}| z\rangle$ is the "classical" distribution function associated to the density matrix $\hat{\rho}$ of the system. The function $\mu(x, p)$ is normalized in the fashion $\int(d p d x / 2 \pi \hbar) \mu(x, p)$ $\equiv \int\left(d x^{\prime} d p^{\prime} / \pi\right) \mu\left(x^{\prime}, p^{\prime}\right)=1$, and is often referred to as the Husimi distribution [13]. It is of particular interest to discuss the equilibrium case as represented by Gibbs' canonical distribution, where the "thermal" density matrix is given by $\hat{\rho}$ $=Z^{-1} e^{-\beta H} . Z=\operatorname{Tr}\left(e^{-\beta H}\right)$ is the partition function and $\beta$ $=1 / k T, T$ being the temperature and $k$ the Boltzmann constant, to be set equal to unity hereafter. Specializing things for the $\mathrm{HO}$, with eigenstates $|n\rangle$ associated to the eigenenergies $E_{n}=\hbar \omega\left(n+\frac{1}{2}\right)$, one has $\langle z|\hat{\rho}| z\rangle=(1 / Z) \sum_{n} e^{-\beta \hat{H}}|\langle z \mid n\rangle|^{2}$ with $|\langle z \mid n\rangle|^{2}=\left(|z|^{2 n} / n !\right) e^{-|z|^{2}}$, so that the distribution $\mu$ reads [3]

$$
\mu(x, p)=\langle z|\hat{\rho}| z\rangle=\left(1-e^{-\beta \hbar \omega}\right) e^{-\left(1-e^{-\beta \hbar \omega}\right)|z|^{2}},
$$

and the Wehrl information (3), after integration over all phase space, turns out to be

$$
I_{W}=1-\ln \left(1-e^{-\beta \hbar \omega}\right) .
$$

Note that $x^{\prime}, y^{\prime}$ in Eq. (1) have been chosen so as to obtain the following result. First define (1) $e_{|z|}^{2}(\beta, \omega) \equiv e_{|z|}^{2}$, (2) $J_{2}$ $=(1 / \pi) \int d x^{\prime} d p^{\prime} \mu\left(x^{\prime}, p^{\prime}\right)|z|^{2}, \quad$ and $=(1 / \pi) \int d x^{\prime} d p^{\prime} \mu\left(x^{\prime}, p^{\prime}\right)|z|$. Then

$$
\begin{aligned}
e_{|z|}^{2} & =J_{2}-J^{2}=\left\langle|z|^{2}\right\rangle_{\mu}-\langle|z|\rangle_{\mu}^{2}=\int \frac{d x^{\prime} d p^{\prime}}{\pi} \mu\left(x^{\prime}, p^{\prime}\right)\left(x^{\prime 2}+p^{\prime 2}\right) \\
& =\left(1-e^{-\beta \hbar \omega}\right)^{-1} .
\end{aligned}
$$

We write down now, for future reference, well-known $\mathrm{HO}$ expressions for the entropy $S$, the mean energy $U$, the specific heat $C_{V}$, and $Z$, respectively. $[14,15]$.

$$
\begin{gathered}
S=\beta \frac{\hbar \omega}{e^{\beta \hbar \omega}-1}-\ln \left\{1-e^{-\beta \hbar \omega}\right\}, \\
U=\hbar \omega\left[\frac{1}{2}+\frac{1}{e^{\beta \hbar \omega}-1}\right], \\
C_{V}=-\beta^{2}(\partial U / \partial \beta)_{V}=\left[\frac{\hbar \omega \beta}{e^{\beta \hbar \omega}-1}\right]^{2} e^{\beta \hbar \omega}, \\
\ln Z=-\beta \frac{\hbar \omega}{2}-\ln \left\{1-e^{-\beta \hbar \omega}\right\} .
\end{gathered}
$$

\section{FISHER'S INFORMATION MEASURE}

One important information measure is that advanced by Fisher in the 1920s (a detailed study can be found in Refs. $[4,5])$. Let us consider a system that is specified by a physical parameter $\theta$, while $\mathbf{x}$ is a stochastic variable $\left(\mathbf{x} \in \operatorname{Re}^{N}\right)$ and $f_{\theta}(\mathbf{x})$ the probability density for $\mathbf{x}$, which depends on the parameter $\theta$. An observer makes a measurement of $\mathbf{x}$ and has to best infer $\theta$ from this measurement, calling the resulting estimate $\tilde{\theta}=\tilde{\theta}(\mathbf{x})$. One wonders how well $\theta$ can be determined. Estimation theory [16] asserts that the best possible estimator $\tilde{\theta}(\mathbf{x})$, after a very large number of $\mathbf{x}$ samples is examined, suffers a mean-square error $e^{2}$ from $\theta$ that obeys a relationship involving Fisher's $I$, namely $I e^{2}=1$, where the Fisher's information measure $I$ is of the form

$$
I(\theta)=\int d \mathbf{x} f_{\theta}(\mathbf{x})\left\{\frac{\partial \ln f_{\theta}(\mathbf{x})}{\partial \theta}\right\}^{2} .
$$

This "best" estimator is called the efficient estimator. Any other estimator must have a larger mean-square error. The only proviso to the above result is that all estimators be unbiased, i.e., satisfy $\langle\tilde{\theta}(\mathbf{x})\rangle=\theta$. Thus, Fisher's information measure has a lower bound, in the sense that, no matter what parameter of the system we choose to measure, $I$ has to be larger than or equal to the inverse of the mean-square error associated with the concomitant experiment. This result, $I e^{2} \geqslant 1$, is referred to as the Cramer-Rao (CR) bound [5]. A particular $I$ case is of great importance: that of translation families [5,6], i.e., distribution functions (DF) whose form does not change under $\theta$ displacements. These DF are shift invariant (in the manner of Mach, no absolute origin for $\theta$ ), and for them Fisher's information measure adopts a somewhat simpler appearance [5]:

$$
I(\text { shift invariant })=\int d \mathbf{x} f(\mathbf{x})\left\{\frac{\partial \ln f(\mathbf{x})}{\partial \mathbf{x}}\right\}^{2} .
$$

Fisher's measure is additive [5]; if $x$ and $p$ are independent variables, $I(x+p)=I(x)+I(p)$. Note that for $\theta \equiv \tau$ $=(x, p)$ (a point in phase space), we face a shift-invariance situation. Since in defining $z$ in terms of the variables $x$ and $p$, these are scaled by their respective variances (cf. above the definition of $\langle z\rangle$ ), the Fisher measure associated to the probability distribution $\mu(x, p) \equiv \mu(\tau)$ will be of the form [6]

$$
I_{\tau}=\int \frac{d p d x}{2 \pi \hbar} \mu(x, p) \mathcal{A},
$$

with

$$
\mathcal{A}=\sigma_{x}^{2}\left[\frac{\partial \ln \mu(x, p)}{\partial x}\right]^{2}+\sigma_{p}^{2}\left[\frac{\partial \ln \mu(x, p)}{\partial p}\right]^{2} .
$$

Given the $\mu$ expression (4), $I_{\tau}$ becomes

$$
I_{\tau}=1-e^{-\beta \hbar \omega}
$$

which, in view of Eq. (6) immediately yields

$$
I_{\tau} e_{|z|}^{2}(\beta, \omega)=1(\mathrm{CR} \text { bound reached }) .
$$

We realize at this point that the Fisher measure built up with Husimi distributions is to be best employed to estimate "phase-space position" $|z|$. Further, efficient estimation is possible for all temperatures, a rather significant result. Comparison with Eq. (5) allows one now to write 


$$
I_{W}=1-\ln \left[I_{\tau}\right] \Rightarrow I_{W}+\ln \left[I_{\tau}\right]=1 .
$$

Since both $I_{W}$ and $I_{\tau}$ are positive-definite quantities, Eq. (14) tells us that they are complementary informational quantities, what one of them gains, the other loses. Following Anderson and Halliwell [3], let us analyze now the high and low temperature limits. When the temperature tends to zero $(\beta \rightarrow \infty), I_{\tau} \approx 1$, its maximum possible value, since we know that the ground state will be the only one to be populated. If, on the other hand, the temperature tends to infinity $(\beta \rightarrow 0)$, then $I_{\tau} \approx \beta \hbar \omega$ and tends to zero because we know beforehand that, in the limit, all energy levels will be populated in uniform fashion. The uniform distribution is that of maximum ignorance [17]. The range of $I_{\tau}$ is $[0,1]$, that of $I_{W}$ is $[1, \infty]$. Replacing $I_{\tau}$ into Eq. (7) we note that

$$
I_{\tau}=\frac{e^{-(1 / 2) \beta \hbar \omega}}{Z},
$$

so that it coincides with the canonical-ensemble probability for finding the system in its ground state.

\section{FISHER, INVERSE TEMPERATURE, AND THERMODYNAMICS' THIRD LAW}

Consider now the general definition (8) of Fisher's information measure in terms of the DF $\mu(x, p)$ :

$$
I_{\beta}=\int \frac{d p d x}{2 \pi \hbar} \mu(x, p)\left(\frac{\partial \ln \mu(x, p)}{\partial \beta}\right)^{2},
$$

with $\beta \equiv \theta$ being the parameter to be estimated. Since

$$
\frac{\partial \ln \mu(x, p)}{\partial \beta}=\frac{\hbar \omega}{e^{\beta \hbar \omega}-1}\left[1-\left(1-e^{-\beta \hbar \omega}\right)|z|^{2}\right],
$$

one readily ascertains that (i) the $\mu$ mean value of Eq. (17) vanishes, and (ii)

$$
I_{\beta}=\left[\frac{\hbar \omega}{e^{\beta \hbar \omega}-1}\right]^{2} \quad\left(T=[0, \infty] \rightarrow I_{\beta}=[0, \infty]\right),
$$

which, in view of Eq. (7), entails

$$
I_{\beta}=\frac{e^{-\beta \hbar \omega}}{\beta^{2}} C_{V} .
$$

Reflection upon the $I_{\beta}$ range (18) might lead one to conclude that it constitutes a Fisher manifestation of thermodynamics' third law. Not only Shannon's measure, but also Fisher's (for the $\mathrm{HO}$, at least) vanishes at zero temperature. Replacing now Eqs. (12) and (18) into the entropy expression [cf. Eq. (7)] we immediately arrive at the relation

$$
S=\beta \sqrt{I_{\beta}}-\ln I_{\tau} .
$$

The HO entropy can be expressed as the sum of two terms: one associated with the Fisher information $I_{\beta}$ and the other with the Fisher information for translation families $I_{\tau}$ corresponding to the phase-space variables $(p, x)$. Using Eq. (7) we also have

$$
\ln I_{\tau}=-\beta \frac{\hbar \omega}{2}-\ln Z=-\left[\beta E_{g S}+\ln Z\right],
$$

with $E_{g s}$ denoting the ground state energy. Thus,

$$
S=\beta\left[\frac{\hbar \omega}{2}+\sqrt{I_{\beta}}\right]+\ln Z,
$$

which is to be compared to the well-known canonicalensemble general expression connecting $S$ and the mean energy $U[14]$,

$$
S=\ln Z+\beta U,
$$

we see that $I_{\beta}$ is related to the excited spectrum contribution to $U$ while $I_{\tau}$ is to be linked to the partition function. We will look now for a new connection between Fisher's measures $I_{\tau}$ and $I_{\beta}$. From Eq. (18) it is possible to rewrite $I_{\beta}$ in the form

$$
I_{\beta} \equiv\left(\frac{\hbar \omega e^{-\beta \hbar \omega}}{1-e^{-\beta \hbar \omega}}\right)^{2},
$$

and therefore

$$
I_{\tau} \sqrt{I_{\beta}}=\hbar \omega e^{-\beta \hbar \omega}=-\frac{\partial\left[e^{-\beta \hbar \omega}\right]}{\partial \beta},
$$

i.e, the product on the left-hand side is the $\beta$ derivative of the Boltzmann factor (constant energywise) at the inverse tem-

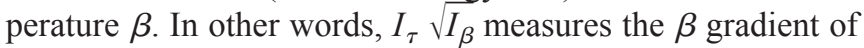
the Boltzmann factor.

\section{UNCERTAINTIES}

We focus attention now on the actual phase-space variables $x, p$ (not on $x^{\prime}, p^{\prime}$ ), and start with the obvious results $\langle x\rangle_{\mu}=\langle p\rangle_{\mu}=0$. We immediately find

$$
\left(\Delta_{\mu} x\right)^{2}=\left\langle x^{2}\right\rangle_{\mu}=\int \frac{d p d x}{2 \pi \hbar} x^{2} \mu(x, p)=\frac{2 \sigma_{x}^{2}}{1-e^{-\beta \hbar \omega}} .
$$

In a similar vein

$$
\left(\Delta_{\mu} p\right)^{2}=\left\langle p^{2}\right\rangle_{\mu}=\frac{2 \sigma_{p}^{2}}{1-e^{-\beta \hbar \omega}},
$$

which entails

$$
\Delta_{\mu} \equiv \Delta_{\mu} x \Delta_{\mu} p=\frac{\hbar}{1-e^{-\beta \hbar \omega}}=\frac{\hbar}{I_{\tau}} \Rightarrow I_{\tau} \Delta_{\mu}=\hbar .
$$

We reconfirm thus the already mentioned fact that phasespace "location" is possible, with Husimi distributions (HPDFs) only up to $\hbar$. This is to be compared to the uncertainties evaluated in a purely quantal fashion without using HPDFs. This is made by recourse to the virial theorem [14], which entails both (i) $U=m \omega^{2}\left\langle x^{2}\right\rangle$ and (ii) $U=\left\langle p^{2}\right\rangle / m$ [cf. Eq. (7)]. From these we easily deduce

$$
\left\langle x^{2}\right\rangle=\sigma_{x}^{2} \frac{e^{\beta \hbar \omega}+1}{e^{\beta \hbar \omega}-1} \Rightarrow\left\langle x^{2}\right\rangle_{\mu}=\frac{2\left\langle x^{2}\right\rangle}{1+e^{-\beta \hbar \omega}}
$$

and 


$$
\left\langle p^{2}\right\rangle=\sigma_{p}^{2} \frac{e^{\beta \hbar \omega}+1}{e^{\beta \hbar \omega}-1} \Rightarrow\left\langle p^{2}\right\rangle_{\mu}=\frac{2\left\langle p^{2}\right\rangle}{1+e^{-\beta \hbar \omega}} .
$$

Consequently,

$$
\Delta x \Delta p=\frac{\hbar}{2} \frac{e^{\beta \hbar \omega}+1}{e^{\beta \hbar \omega}-1} \Rightarrow \Delta_{\mu}=\frac{2 \Delta x \Delta p}{1+e^{-\beta \hbar \omega}} .
$$

As $\beta \rightarrow \infty, \Delta_{\mu}$ is twice the minimum quantum value for $\Delta x \Delta p$, and $\Delta_{\mu} \rightarrow \hbar$, the "minimal" phase-space cell. The quantum and semiclassical results do coincide at a very high temperature, though. Finally, with the help of Refs. [24,25], one readily can recast Heisenberg's uncertainty relation as a function of both frequency and temperature in the fashion

$$
F(\beta, \omega)=\Delta x \Delta p=\frac{\hbar}{2 I_{\tau}}\left(1+e^{-\beta \hbar \omega}\right)=\frac{1}{2}\left[\frac{\hbar}{I_{\tau}}+\frac{\sqrt{I_{\beta}}}{\omega}\right],
$$

so that, for $T$ varying in $[0, \infty]$, the range of possible $\Delta x \Delta p$ values is $[\hbar / 2, \infty]$. Equation (32) is a "Heisenberg-Fisher" thermal uncertainty (TU) relation (for a discussion of the TU concept, see, for instance, Refs. [8,18,19]. $F(\beta, \omega)$ grows with $I_{\beta}$ and diminishes with $I_{\tau}$. Note that, for fixed uncertainty $F(\beta, \omega)=$ const, $I_{\tau}$ and $I_{\beta}$ play "parallel" roles: improving temperature-estimation performance (in the sense that $I_{\beta}$ grows) also enhances that of phase-space location ( $I_{\tau}$ has to grow as well), and vice versa.

\section{CONCLUSIONS}

We have explored in this work connections between canonical-ensemble quantities and two FIMs, associated to the estimation of, respectively, (i) phase-space location $\left(I_{\tau}\right)$ and temperature $\left(I_{\beta}\right)$. Our most important result is, perhaps, to show that there exists a "Fisher-associated" third law of thermodynamics (at least for the HO). From a pure information-theoretic viewpoint, we have, in addition, advanced several results, namely, (1) a connection between Wehrl's entropy and $I_{\tau}$ [cf. Eq. (14)], (2) an interpretation of $I_{\tau}$ as the HO's ground state occupation probability [cf. Eq. (15)], (3) an interpretation of $I_{\beta}$ proportional to the HO's specific heat [cf. Eq. (19)], (4) the possibility of expressing the HO's entropy as a sum of two terms, one for each of the above FIM realizations [cf. Eq. (20)], (5) a new form of Heisenberg's uncertainty relations in Fisher terms [cf. Eq. (32)], (6) efficient $|z|$ estimation can be achieved with $I_{\tau}$ at all temperatures, as the minimum Cramer-Rao value is always reached [cf. Eq. (13)].

These results are, of course, restricted to the harmonic oscillator. However, this is such an important system that $\mathrm{HO}$ insights usually have a wide impact, as the $\mathrm{HO}$ constitutes much more than a mere example. Nowadays it is of particular interest for the dynamics of bosonic or fermionic atoms contained in magnetic traps [20-22] as well as for any system that exhibits an equidistant level spacing in the vicinity of the ground state, like nuclei or Luttinger liquids.
[1] A. Wehrl, Rep. Math. Phys. 16, 353 (1979).

[2] E. H. Lieb, Commun. Math. Phys. 62, 35 (1978).

[3] A. Anderson and J. J. Halliwell, Phys. Rev. D 48, 2753 (1993).

[4] B. R. Frieden and B. H. Soffer, Phys. Rev. E 52, 2274 (1995).

[5] B. R. Frieden, Physics from Fisher Information (Cambridge University Press, Cambridge, England, 1998).

[6] F. Pennini, A. R. Plastino, and A. Plastino, Physica A 258, 446 (1998).

[7] B. R. Frieden, A. Plastino, A. R. Plastino, and H. Soffer, Phys. Rev. E 60, 48 (1999).

[8] F. Pennini, A. Plastino, A. R. Plastino, and M. Casas, Phys. Lett. A 302, 156 (2002).

[9] A. R. Plastino and A. Plastino, Phys. Rev. E 54, 4423 (1996).

[10] A. Plastino, A. R. Plastino, and H. G. Miller, Phys. Lett. A 235, 129 (1997).

[11] J. R. Klauder and B. S. Skagerstam, Coherent States (World
Scientific, Singapore, 1985).

[12] J. Schnack, Europhys. Lett. 45, 647 (1999).

[13] K. Husimi, Proc. Phys. Math. Soc. Jpn. 22, 264 (1940).

[14] R. K. Pathria, Statistical Mechanics (Pergamon Press, Exeter, 1993).

[15] C. Cohen-Tannoudji, B. Diu, and F. Lalo, Quantum Mechanics (Hermann, Paris, 1977).

[16] H. Cramer, Mathematical Methods of Statistics (Princeton University Press, Princeton, NJ, 1946).

[17] A. Katz, Principles of Statistical Mechanics, The Information Theory Approach (Freeman, San Francisco, 1967).

[18] B. Mandelbrot, Ann. Math. Stat. 33, 1021 (1962).

[19] F. Pennini and A. Plastino, Physica A 334, 132 (2004).

[20] M. H. Anderson et al., Science 269, 198 (1995).

[21] K. B. Davis et al., Phys. Rev. Lett. 75, 3969 (1995).

[22] C. C. Bradley, C. A. Sackett, and R. G. Hulet, Phys. Rev. Lett. 78, 985 (1997). 